\title{
THE APRIL MEETING OF THE SOCIETY AT CHICAGO.
}

THE thirty-seventh regular meeting of the Chicago Section, being the sixth regular meeting of the Society at Chicago, was held at the University of Chicago, on Friday and Saturday, April 21 and 22. The meeting was attended by about sixtyfive persons, among whom were the following forty-six members of the Society:

Professor R. M. Barton, Dr. Josephine E. Burns, Professor D. F. Campbell, Professor R. D. Carmichael, Dr. E. W. Chittenden, Dr. G. R. Clements, Professor H. E. Cobb, Professor D. R. Curtiss, Professor S. C. Davisson, Dr. W. W. Denton, Professor L. E. Dickson, Professor A. Dresden, Professor W. B. Ford, Professor Tomlinson Fort, Professor A. B. Frizell, Professor A. S. Hathaway, Professor E. R. Hedrick, Dr. Cora B. Hennel, Professor W. C. Krathwohl, Dr. W. V. Lovitt, Professor A. C. Lunn, Professor W. D. MacMillan, Dr. T. E. Mason, Professor G. A. Miller, Professor E. H. Moore, Professor E. J. Moulton, Professor F. R. Moulton, Dr. A. L. Nelson, Professor C. I. Palmer, Dr. A. Pell, Professor H. L. Rietz, Professor W. H. Roever, Professor D. A. Rothrock, Miss I. M. Schottenfels, Mr. A. R. Schweitzer, Professor J. B. Shaw, Professor C. H. Sisam, Professor H. E. Slaught, Professor R. B. Stone, Professor E. J. Townsend, Professor A. L. Underhill, Professor E. B. VanVleck, Professor E. J. Wilczynski, Professor K. P. Williams, Dr. C. H. Yeaton, Professor J. W. A. Young.

The sessions were presided over by Professor W. B. Ford, chairman of the Section, relieved during the session of Saturday forenoon by Professor E. R. Hedrick, vice-president of the Society. Forty-four persons were present at the dinner on Friday evening.

The following papers were presented at this meeting:

(1) Professor D. M. Sмiтн: "Jacobi's condition for the problem of Lagrange in the calculus of variations."

(2) Professor C. H. Sisam: "On a configuration on certain surfaces." 
(3) Professor T. R. Running: "A new method for deriving weir formulas."

(4) Professor ARNold Emch: "A new configuration on an elliptic cubic and its group of order 16."

(5) Professor L. L. Dines: "A characteristic property of self-projective curves."

(6) Dr. A. L. Nelson: "Plane nets with equal invariants."

(7) Dr. A. L. NeLson: "Quasi-periodic asymptotic plane nets."

(8) Dr. E. W. Chittenden: "A theorem in general analysis."

(9) Dr. E. W. Chittenden: "On the equivalence of écart and voisinage."

(10) Professor R. D. Carmichael: "On the asymptotic character of functions defined by series of the form $\Sigma c_{n} g(x+n) . "$

(11) Professor W. D. MacMillan: "A theorem relating to irrational numbers."

(12) Professor W.D. MacMrllan: "A reduction of certain differential equations of the second order to algebraic types."

(13) Professor Tomuinson Font: "A class of developments in orthogonal functions."

(14) Dr. R. L. Borgen: "On the Cauchy-Goursat theorem."

(15) Professor H. T. Burgess: "A practical method for determining elementary divisors."

(16) Dr. J. O. Hassler: "Plane nets periodic of period 3 under the Laplacian transformation."

(17) Professor A. S. Hathaway: "The expansion, in terms of the coefficients of an equation, of the homogeneous products of the roots as a whole, and when restricted to $k$ roots in each term."

(18) Professor W. B. Ford: "A theorem in the calculus of residues."

(19) Professor G. A. Miller: "Graphical method of finding the possible sets of independent generators of an abelian group."

(20) Professor A. B. Frizell: "Postulates of continuity for arithmetic."

(21) Professor K. P. Wilurams: "Concerning Hill's derivation of the Lagrange equations of motion."

(22) Professor Henry Blumberg: "On convex functions." 
(23) Professor Henry Blumberg: "On a theorem of Kempner concerning transcendental numbers."

(24) Professor E. B. VAN VleCK: "On the composition of non-loxodromic substitutions."

(25) Mr. Tobias Dantzig: "A geometrical treatment of plane transformations."

(26) Professor E. H. Moore: "On a definition of the concept: limit of a function."

(27) Mr. A. Elmendorf: "A differentiating machine."

(28) Mr. A. M. HaRding: "On certain loci projectively connected with a given plane curve."

(29) Miss Pauline Sperry: "Properties of a certain projectively defined two-parameter family of curves on a general surface."

(30) Dr. C. H. Yeaton: "Surfaces characterized by certain special properties of their directrix congruences."

Dr. Hassler, Mr. Harding and Miss Sperry were introduced by Professor Wilczynski, Mr. Dantzig by Professor Davisson, and Mr. Elmendorf by Professor Dresden. The papers of Professors Smith, Running, Dines, Burgess and Blumberg, and Dr. Borger were read by title.

Abstracts of the papers follow below. The abstracts are numbered to correspond to the titles in the list above.

1. For the problem of Lagrange, in the calculus of variations, the Euler rule, Weierstrass condition, and corner point condition are obtainable without the use of the second variation. The Jacobi condition, however, is derived by the use of complicated transformations of the second variation or with the exclusion of important special cases. In the paper of Professor D. M. Smith it is shown that the well-known necessary condition for a minimum-i. e., that the second variation must be positive or zero for every set of admissible variations-implies a problem of Lagrange of precisely the same type as the original problem. An application of the Euler rule, Weierstrass condition, and corner point condition to this new problem leads to simple and inclusive proofs of the Legendre and Jacobi conditions for the original problem. The paper will appear in the Transactions.

2. Professor Sisam's paper appeared in full in the May Bulletin. 
3. The following method for deriving the formulas for the rate of flow of water over weirs avoids the necessity of a large number of experiments and can be applied to weirs of any head.

A tank having a constant horizontal cross-section has a weir fitted with a gate which can easily be removed to allow the flow of water. A chronograph connected electrically with a float and also with a vibrating pendulum records time to hundredths of a second and head to thousandths of a foot. Professor Running experimented with a right-angled notch weir, head from $2 \mathrm{ft}$. to $1.7 \mathrm{ft}$., and obtained for the law connecting time and head

$$
T=-23+62.2 H^{-1.47}
$$

$T$ and $H$ must satisfy the relation

$$
Q d T=-S d H,
$$

where $Q$ represents the rate of outflow and $S$ the horizontal cross-section of the tank. From these two equations the formula for the weir was found to be

$$
Q=2.52 H^{2.47}
$$

This represents quite closely the results of experiments performed by other methods.

The above is part of a paper to be published jointly with Professor King.

4. It is well known that a plane cubic may be generated in an infinite number of ways by projective quadratic pencils of straights with a self-corresponding element. The vertices of these pencils are points of a Steinerian couple. A Steinerian quadruple on an elliptic cubic is formed by the points of tangency of the four tangents from a point of the elliptic cubic to the same cubic. The quadruple contains six couples, and, consequently, six projectivities of quadratic pencils may be formed which may be written in 12 different ways, and which all generate the same cubic. Applying a certain cyclic process to the equations of these projectivities and making use of a certain theorem in algebra, Professor Emch obtains a configuration of 48 straights, which in triplets are concurrent 
in 16 points. From one triplet all the others are obtained by the substitutions of a group of order 16 with 15 subgroups of order 2. The paper will be published in the Annals of Mathematics.

5. Self-projective curves, or $W$-curves, were defined by Klein and Lie to be those curves which admit infinitesimal projective transformations into themselves. In this paper, Professor Dines determines for these curves a simple characteristic property somewhat similar to the Steinerian definition of conics. The paper will appear in the Annals of Mathematics.

6. Wilczynski has treated the projective differential geometry of plane nets, as well as that of curved surfaces. Dr. Nelson sets up, in this paper, a perspective connection between these two theories by means of the following theorem of Koenigs: The asymptotic curves of a surface are projected from a fixed point on a fixed plane in a net with equal LaplaceDarboux invariants, and conversely, a plane net with equal invariants may be considered as the perspectives, from a fixed point, of the asymptotic curves of a surface. The paper will be offered for publication to the Palermo Rendiconti.

7. Dr. Nelson's second paper appears in the present number of the BULLETIN.

8. Dr. Chittenden completes the proof of the theorem: Every class $\mathfrak{M}$ with the composite property $D_{1} K_{12} \Delta$ has the property $K_{12 *}$, and $\mathfrak{M}_{*}$ has the composite property

$$
L C D_{1} A \Delta K_{12} K_{12 *} \text {. }
$$

The proof is accomplished with the aid of a theorem of Professor A. D. Pitcher which differs from the present theorem in that the hypothesis contains the additional property $B_{3}$. $B_{3}$ is a special property defined in terms of $\Delta$ and boundedness from zero.

Professor Pitcher in a discussion of the complete existential theory of the eight properties $L, C, D, A, K_{1}, K_{2}, \Delta, K_{12 *}$, left unsettled the question of the existence of three composite properties; viz.,

$$
\begin{gathered}
L-C-D A \Delta K_{1} K_{2}-K_{12 *} ; \quad L C-D A \Delta K_{1} K_{2}-K_{12 *} ; \\
L C-D-A \Delta K_{1} K_{2}-K_{12 *} .
\end{gathered}
$$


The theorem of this paper shows that the first two are nonexistent, and that the third is non-existent for any system $\left(\mathfrak{P} ; \Delta ; \mathfrak{M}^{D_{1}}\right)$. This extends the results obtained by Professor Pitcher who showed that the three properties were nonexistent for any system $\left(\mathfrak{B} ; \Delta ; \mathfrak{M}^{D_{1} B_{8}}\right)$. It is understood that the development $\Delta$ is finite.

9. Hahn has shown that if a voisinage $V(a, b)$ is defined for a class $\mathfrak{Q}$ containing at least two elements, then for every element $q$ of $\mathfrak{Q}$ there exists a continuous function $\mu^{q}$, vanishing only at $q$ and assuming values between zero and one. Dr. Chittenden modifies the existence proof of Hahn and secures a type of uniformity among the functions $\mu^{q}$. Defining $(a, b)$ as the least upper bound of $\left|\mu^{q}(a)-\mu^{q}(b)\right|$ for all elements $q$ of $\mathfrak{Q}$, it appears that $(a, b)$ is an écart and is defined for every pair of elements of $\mathfrak{Q}$. By means of the uniformity mentioned it is shown that $L_{n}\left(a_{n}, a\right)=0$ implies $L_{n} V\left(a_{n}, a\right)=0$ and that $L_{n} V\left(a_{n}, a\right)=0$ implies $L_{n}\left(a_{n}, a\right)=0$. Hence voisinage is equivalent to écart. This result was anticipated by Fréchet on the ground that no theorem was known for an écart which had not been established for a voisinage. This paper will be offered to the Transactions for publication.

10. In a previous paper (read before the Society at the recent meeting in Columbus) Professor Carmichael laid the foundations of a general theory of the series

$$
\Omega(x)=\sum_{n=0}^{\infty} c_{n} g(x+n),
$$

where $g(x)$ is a function having, in a sector $V$, the Poincaré asymptotic representation

$$
g(x) \sim x^{P(x)} e^{\ell(x)}\left(1+\frac{a_{1}}{x}+\frac{a_{2}}{x^{2}}+\cdots\right),
$$

$P(x)$ and $Q(x)$ being polynomials in $x$. In the present paper he shows that a function $\Omega(x)$ defined by the foregoing series satisfies the relations

$$
\begin{aligned}
\lim \{g(x+s)\}^{-1}\left\{\Omega(x)-\sum_{n=0}^{s} c_{n} g(x+s)\right\}=0 & \\
& (s=0,1,2, \cdots),
\end{aligned}
$$

provided that $x$ approaches infinity in an appropriately deter- 
mined sector $\bar{V}$ lying in $V$. Guided by this result he introduces a generalization of Poincarés notion of asymptotic representation and discusses the character of this generalized representation, especially in relation to that of Poincaré.

11. In celestial mechanics, series occur very frequently which contain denominators having factors of the form $(i-j \gamma)$ where $\gamma$ is an irrational number; $i$ and $j$ are integers which take all possible values. The study of series in which this phenomenon occurs has led Professor MacMillan to the following theorem:

If $\gamma$ is a positive number, and if $p_{n} / n$ is a rational fraction such that $\left|p_{n}-n \gamma\right| \leqq \frac{1}{2}$, and if

$$
G_{n}=\left(\prod_{k=1}^{n}\left|p_{k}-k \gamma\right|\right)^{1 / n}
$$

is the geometric mean of the first $n$ of the quantities $\left|p_{k}-k \gamma\right|$, then the limit of $G_{n}$, as $n$ increases without limit, is zero if $\gamma$ is rational, and is equal to $1 / 2 e$, where $e=2.71828 \cdots$ is the Naperian base, if $\gamma$ is an irrational number which satisfies the condition that

$$
a_{n+1} \leqq M q_{n}\left(q_{n}+1\right) \cdots\left(q_{n}+s\right),
$$

where $\gamma$ expressed as a continued fraction is

$$
\gamma=a_{1}+\frac{1}{a_{2}+\frac{1}{a_{3}}+},
$$

where $q_{n}$ is the denominator of the $n$th principal convergent, and $s$ is any assigned positive integer independent of $n$. If $\gamma$ is an irrational number which does not satisfy this condition then $G_{n}$ for large values of $n$ oscillates between zero and 1/2e.

This paper will appear in the American Journal of Mathematics.

12. In two former papers Professor MacMillan has shown that certain differential equations can be reduced by a lineartranscendental substitution to algebraic forms. The first of these papers dealt with a "general case," the second with equations of the first order.

The exceptions to the general case include most of the equations of dynamics, and therefore these equations require 
further study. The present investigation takes up differential equations of the second order

$$
\frac{d x_{i}}{d t}=\alpha_{i} x_{i}+\text { terms of higher degree } \quad(i=1,2),
$$

where all of the coefficients of the right members are constants, and where the $\alpha_{i}$ considered as points in the complex plane lie on a straight line through the origin and on opposite sides of the origin. If these conditions on the $\alpha_{i}$ are not satisfied the equations belong to the general case.

If the ratio $\alpha_{2} / \alpha_{1}$ is an irrational number of a certain type (which includes all irrational algebraic numbers) then the differential equations can be reduced by a linear-transcendental substitution to its linear terms, and the substitution is convergent if the differential equations are convergent.

If the ratio $\alpha_{2} / \alpha_{1}$ is rational there is no essential restriction in supposing that $\alpha_{1}=+1, \alpha_{2}=-1$. In this case the differential equations cannot, in general, be reduced to their linear terms. They can however be reduced to a set of differential equations which are algebraic, and which are easily integrable. The convergence of the substitution is assured if the equations are canonical

$$
x_{1}^{\prime}=\frac{\partial H}{\partial x_{2}}, \quad x_{2}^{\prime}=-\frac{\partial H}{\partial x_{1}},
$$

where $H$ is a convergent power series in $x_{1}$ and $x_{2}$. In certain other cases, however, the substitution may diverge even though the differential equations converge. The paper will be offered to the Transactions.

13. Professor Fort's paper considers the development of an arbitrary function $f(x)$ in infinite series of solutions of

$$
\frac{d}{d x}\left[k(x) y^{\prime}(x)\right]-\left[\lambda^{2} g(x)+l(x)\right] \dot{y}=0,
$$

satisfying the conditions (2) $y(0)=y(2 \pi), y^{\prime}(0)=y^{\prime}(2 \pi)$; and secondly in terms of solutions satisfying the conditions (3) $y(0)=-y(2 \pi), y^{\prime}(0)=-y^{\prime}(2 \pi)$. The principal results are given in the following theorem.

Let $(k) x$ and $g(x)$ be positive at all points and have second derivatives integrable from 0 to $2 \pi$. Let $l(x)$ have a first 
derivative integrable from 0 to $2 \pi$ and let $f(x)$ itself be integrable from 0 to $2 \pi$. Let moreover $g(0) k(0)=g(2 \pi) k(2 \pi)$ and $[g(0) k(0)]^{\prime}=[g(2 \pi) k(2 \pi)]^{\prime}$. Then the development corresponding to $f(x)$ in terms of solutions of (1) satisfying (2) (more accurately described in the paper) converges at any particular point of (4) $0 \leqq x \leqq 2 \pi$, when and only when the Fourier series for $f(x)$ converges at that point and to the same value. It converges uniformly over the whole interval or any subinterval when and only when this is true of the Fourier series and diverges to $\infty$ or $-\infty$ at any particular point when and only when this is true of the Fourier series. Moreover it is summable by the method of the arithmetic mean when and only when the same thing is true of the Fourier series and to the same value.

Secondly: The development in terms of the solutions of (1) satisfying (3) bears the same relation to the development of $f(x)$ in terms of $\sin [(2 n+1) / 2] x$ and $\cos [(2 n+1) / 2] x, n=0$, $1,2, \cdots$ as is borne by the series just discussed to the Fourier series.

14. In this paper Dr. Borger shows that if the two functions $U(x, y), V(x, y)$ satisfy the following conditions in a region $R$ :

(1) $U$ and $V$ are continuous in $(x, y)$,

(2) $U_{x}^{\prime}, V_{x}^{\prime}, U_{y}^{\prime}, V_{y}^{\prime}$ exist and are finite,

(3) $U_{x}^{\prime}=V_{y}^{\prime} ; U_{y}^{\prime}-V_{x}^{\prime}$,

(4) $U$ and $V$ possess proper total differentials;

then $U$ and $V$ are analytic functions of the real variables $x, y$.

An immediate corollary is:

If $w=f(z)=U(x, y)+i V(x, y)$ possess a finite derivative at every point of a region $R$ :

(a) This derivative is a continuous function of $z$.

(b) All the derivatives of $w$ exist.

(c) $w$ can be represented by a power series in $z$.

This paper will be offered ta the BuLLETIN for publication.

15. In this paper Professor Burgess shows that there exists a very simple relation between the exponents of the elementary divisors of the characteristic matrix $\lambda I+B$ which are connected with the linear factor $\lambda-\alpha$ of the characteristic equation $|\lambda I+B|=0$, and the ranks of the matrices $(\alpha I+B)^{n}$ for $n=1,2,3$, etc. In fact these ranks determine the elementary divisors and conversely. 
[June,

It is also shown that the method is easily applied to any matrix of the form $\lambda A+B$ whose determinant does not vanish identically in $\lambda$. The paper will be published in the Annals of Mathematics.

16. In the projective theory of plane nets, as developed by Wilczynski, the members $y_{1}(u, v), y_{2}(u, v), y_{3}(u, v)$ of any fundamental system of solutions of a completely integrable system of partial differential equations of the form

$$
\begin{aligned}
& y_{u u}=a y_{u}+b y_{v}+c y, \\
& y_{u v}=a^{\prime} y_{u}+b^{\prime} y_{v}+c^{\prime} y, \\
& y_{v v}=a^{\prime \prime} y_{u}+b^{\prime \prime} y_{v}+c^{\prime \prime} y,
\end{aligned}
$$

are interpreted as the homogeneous coordinates of a point $P_{y}$ in a plane, defining a non-degenerate net of plane curves. The invariants and covariants of the system (1) under the transformations $y=\lambda(u, v) \bar{y}$ and $\bar{u}=U(u), \bar{v}=V(v)$ may be interpreted geometrically by certain projective properties of the net. The two covariants $\rho=y_{u}-b^{\prime} y, \sigma=y_{v}-a^{\prime} y$ give rise to the homogeneous coordinates of two points $P_{\rho}$ and $P_{\sigma}$ when we substitute successively for $y$ the values $y_{1}, y_{2}, y_{3}$. As $u$ and $v$ vary $P_{\sigma}$ and. $P_{\rho}$ describe nets called the first and minus first Laplacian transforms of the original net. The minus first Laplacian transform of the $\sigma$-net and the first Laplacian transform of the $\rho$-net both coincide with the original $y$-net.

In this paper Dr. Hassler considers classes of nets such that the first Laplacian transform of the $\sigma$-net is the $\rho$-net and the minus first Laplacian transform of the $\rho$-net is the $\sigma$-net. Such nets are periodic of period 3 under the Laplacian transformation. He establishes necessary and sufficient conditions for such periodicity, determines the form of the coefficients of system (1) for such a net, computes its invariants and covariants, and studies the osculating conics of the curves of the original net and of its Laplacian transforms. The determination of a net of this kind by certain boundary conditions is considered and the following theorem is proved:

Choose an arbitrary triangle $L M N$. Through the point $L$ pass two non-rectilinear but otherwise arbitrary analytic curves $C$ and $C^{\prime}$, tangent to $L N$ and $L M$, respectively, at $L$. Through each of the points $M$ and $N$ pass another such arbi- 
trary curve, $C_{1}{ }^{\prime}$ through $M$ and $C_{-1}$ through $N$, tangent to $M N$ at $M$ and $N$, respectively. Select any conic $M_{1}$ tangent to $L M$ at $M$ and a second conic $N_{-1}$ tangent to $L N$ at $N$. There exists one and only one net which is periodic of period 3 under the Laplacian transformation, which contains the given curves $C$ and $C^{\prime}$, which has $C_{-1}$ corresponding to $C$ and $C_{1}{ }^{\prime}$ corresponding to $C^{\prime}$ as curves of the minus first and first Laplacian transforms respectively, and which, moreover, has $M_{1}$ as the osculating conic of the curve of the first Laplacian transform corresponding to $C$ and $N_{-1}$ as the osculating conic of the curve of the minus first Laplacian transform corresponding to $C^{\prime}$.

This paper will appear in the Palermo Rendiconti.

17. In this paper, Professor Hathaway shows, from the properties of gamma coefficients defined by

$$
(A \alpha B \beta)=(A \alpha+B \beta) \Gamma(\alpha+\beta) / \Gamma(\alpha+1) \Gamma(\beta+1), \text { etc., }
$$

that the function of weight $n$ in the coefficients of the equation

defined by

$$
x^{m}=a_{1} x^{m-1}+\cdots+a_{m}
$$

$$
F(n)=\Sigma\left(A_{1} \alpha_{1} A_{2} \alpha_{2} \cdots A_{n} \alpha_{n}\right) a_{1}^{\alpha_{1}} a_{2}{ }^{\alpha_{2}} \cdots a_{n}{ }^{a_{n}}
$$

(the summation being for all integral solutions of

$$
\left.\alpha_{1}+2 \alpha_{2}+\cdots+n \alpha_{n}=n\right)
$$

is the unique solution of the difference equation

$$
F(n)=a_{1} F(n-1)+\cdots+a_{n-1} F(1)+A_{n} a_{n} .
$$

When $n^{\prime}>m, a_{n^{\prime}}=0$, in the above values of $F(n)$.

Hence, the problem of expanding any symmetric function of the roots is the problem of determining the coefficient $A_{n}$ in its difference equation of the above type. Thus, for $\pi(n)$, the homogeneous products, $A_{n}=1$, i. e., the numerical coefficient of any term in $\pi(n)$ is the multinomial coefficient of its exponents $\left(1 \alpha_{1} 1 \alpha_{2} \cdots 1 \alpha_{n}\right)$.

Also, for the homogeneous products, one root in a term, $\pi_{1}(n)=s_{n}$, by Newton's formulas, $A_{n}=n$. The resultant coefficient of any term $\left(1 \alpha_{1} 2 \alpha_{2} \cdots n \alpha_{n}\right)$ is identical with Waring's.

To determine $A_{n}$ for the homogeneous products, $k$ roots 
at a time, $\pi_{k}(n)$, we have only to consider the equation $x^{n}=1$, and find $A_{n}=(-1)^{k+1}[1(n-k) 1 k]$, a binomial coefficient.

There is a remarkable relation between the homogeneous products of all weights, and those of weight $n$ and all orders $k$. Thus, expanding $F(n)$ in terms of the arbitrary quantities $A$, we obtain

$$
F(n)=A_{1} a_{1} \pi_{n-1}+A_{2} a_{2} \pi_{n-2}+\cdots+A_{n} a_{n} .
$$

Thus we have the table,

\begin{tabular}{|c|c|c|c|c|c|}
\hline & $a_{1} \pi_{n-1}$ & $a_{2} \pi_{n-2}$ & $a_{3} \pi_{n-3}$ & $a_{4} \pi_{n-4}$ & $\cdots$ \\
\hline$\pi(n)=$ & 1 & 1 & 1 & 1 & $\cdots$ \\
\hline$\pi_{1}(n)=$ & 1 & 2 & 3 & 4 & \\
\hline$-\pi_{2}(n)=$ & & 1 & 3 & 6 & \\
\hline$\pi_{3}(n)=$ & & & 1 & 4 & \\
\hline$-\pi_{4}(n)=$ & & & & 1 & \\
\hline$\cdots$ & & & & & \\
\hline
\end{tabular}

The relation may also be written as an identity in an arbitrary variable $x$,

$$
\begin{aligned}
\Sigma(-x)^{k} \pi_{k}(n)+\Sigma(1+x)^{k} a_{k} \pi(n-k)= & \pi(n) \\
& (k=1,2, \cdots, n),
\end{aligned}
$$

and by substituting $-1-x$ for $x$ we see that the relation is symmetrical.

18. Cauchy's integral theorem for functions of a complex variable has often been employed to advantage for the summing of infinite series, such applications belonging to the subject commonly known as "the calculus of residues." After pointing out the limitations naturally present in using this method of summation, Professor Ford's paper proceeds to outline a way by which the fundamental difficulty may be avoided in many cases, and an actual formula for sum is obtained which has a wide range of applicability. The paper will be offered for publication to the BuLletin. 
19. The term independent generators of an abelian group has two distinct meanings. According to one of these meanings the set of operators $s_{1}, s_{2}, \cdots, s_{\lambda}$ is said to be a set of independent generators of the group $G$ provided these operators generate $G$ but no $\lambda-1$ of them generate $G$. According to the other meaning such a set is called a set of independent generators of $G$ if and only if the group generated by every $\lambda-1$ of them has only identity in common with the group generated by the remaining one. The value of $\lambda$ is the same in both cases. When the term independent generator is used with the former meaning it is said to be used in the general sense and when it is used with the latter meaning it is said to be used in the restricted sense. In the general sense the operators which cannot be used as independent generators constitute a subgroup known as the $\phi$-subgroup. In the restricted sense these operators do not always constitute a subgroup.

Professor Miller's results relate to the use of independent generators in the restricted sense. If the order of an abelian group $G$ is $p^{m}, p$ being a prime number, and if we form the quotient group of $G$ with respect to its subgroup $H$ composed of the $p \mathrm{th}$ power of each of its operators, the order of this quotient group being $p^{\lambda}$, then $\lambda$ is the number of the independent generators of $G$. The number of operators of $G$ which can be used as independent generators of lowest order is equal to the number of operators of lowest order in the cosets of $G$ with respect to $H$. The totality of the operators which can be used as independent generators of other orders may be determined similarly.

20. The continuity axioms of Cantor and Weierstrass are essentially assumptions about the existence of limits. Professor Frizell submits a set of postulates based on ideas of order, from which it is easy to deduce both Cantor's assumption and the principle of Archimedes, and therefore also the postulates of Weierstrass and Dedekind. This paper will be offered to the BuLLETIN for publication.

21. Professor Williams's paper appears in the present number of the BuLletin.

22. A real function $f(x)$ uniquely defined in the interval $(a, b)$ 
is said to be "convex," if for every two values $x_{1}$ and $x_{2}$ in the interval $(a, b)$ the inequality

$$
f\left(\frac{x_{1}+x_{2}}{2}\right) \leqq \frac{f\left(x_{1}\right)+f\left(x_{2}\right)}{2}
$$

holds.* From a paper of Bernstein and Doetsch,+ according to which a convex function is either continuous (except possibly at the ends of the interval) or totally discontinuous, it appears that discontinuous convex functions are of relatively minor importance. Let then the continuity of $f(x)$ be assumed henceforth. Jensen has shown $\ddagger$ that the derivative of $f(x)$ may be non-existent in an everywhere dense set of points, but the right-hand derivative (together with the left-hand derivative) always exists. Professor Blumberg proves (among other things): (a) The right-hand derivative of $f(x)$ is a monotone increasing function; conversely, if the right-hand derivative of a continuous function $g(x)$ exists everywhere and is a monotone increasing function, then $g(x)$ is convex. (b) The right-hand derivative of $f(x)$ is continuous on the right at every point of $(a, b)$. (c) The points of $(a, b)$ at which the derivative of $f(x)$ is non-existent form at most a denumerable set (conversely, given a denumerable set $S$, a convex function $f(x)$ may be constructed, such that the derivative of $f(x)$ is existent at every point not in $S$ and non-existent at every point in $S)$. (d) Every continuous convex function is the indefinite integral of a monotone increasing function; conversely, the indefinite integral of a monotone increasing function is convex (and continuous).

The proofs are extremely simple. Moreover, the proofs of most of the theorems (on continuous convex functions) contained in the two papers quoted above are simplified and rendered nearly intuitionally evident; the Cauchy artifice,§ which is at the basis of the proofs of Jensen, Bernstein and Doetsch, may be altogether dispensed with.

23. The theorem in question is as follows: $\|$ The number

$$
f(x)=\sum_{r=0}^{r=\infty} \alpha_{r} x^{r} / a^{c^{r}}
$$

* See Jensen, Acta Math., vol. 30, p. 176.

† Math. Ann., 1915, p. 514.

$¥$ L. c.

\$ See Jensen, 1. c., p. 175.

See Bulletin, Mar., 1915, p. 285. 
is transcendental for every rational (real) $x(\neq 0)$, if $a$ and $c$ are integers $\geqq 2$ and if (1) $\left|\alpha_{r}\right|<M^{r}, M$ arbitrary but fixed, and (2) only a finite number of the $\alpha$ 's vanish. Professor Blumberg shows by means of a modification of Dr. Kempner's method that the condition (2) may be substantially altogether dropped and replaced by the restriction (evidently to be demanded) that $f(x)$ shall not break off after a finite number of terms. Condition (1) may be replaced by the milder condition $\left|\alpha_{r}\right|<M^{r^{k}}, k$ being like $M$ arbitrary but fixed.

25. In this paper Mr. Dantzig considers a continuous plane $n$ to 1 transformation $T$. Let $C$ be an arbitrary curve through a point $P$ and having $t$ for tangent at $P$ and let $C_{1} P_{1}$ be the images of $C$ and $P$, and $t_{1}$ the tangent to $C_{1}$ at $P_{1} ; t$ and $t_{1}$ will meet in a point $\tau$. The locus of the point $\tau$ for the totality of the curves $C$ through $P$ is a conic, which the author calls the indicatrix of $T$ for the point $P$. There exists for each point in the plane such an indicatrix $I$, and the totality of the indicatrices $I$ form a two-parameter system of conics. It is further shown that the behavior of $T$ at a point $P$ is characterized by the indicatrix at $P$. In particular if the totality of the indicatrices form a system of circles or equilateral hyperbolas, the transformation is conformal.

The case of the degeneracy of the indicatrix is further taken up and the intimate relation of the indicatrix to the invariant elements of $T$ is brought out. It is shown that in the case of the identical degeneracy of the indicatrix either $T$ is a pseudo transformation, or there exists an infinity of invariant straight lines enveloping an absolutely invariant curve.

The value of the method for the classification of plane transformations is discussed. Applied to collineations the method furnishes a purely geometrical basis for projective geometry independent of any consideration of anharmonic ratios, elliptic involutions or polar reciprocity.

The author applies this method to a great number of problems in geometry, particularly to the proof of Poincarés last theorem, to the problem of closure and the theory of unicursal plane curves.

26. By means of a remarkably simple generalization of the concept limit of a function Professor E. H. Moore has recently found it possible to define the integration process $J$ in a new 
theory of linear integral equations in general analysis, a general theory having as its guiding instance a body of theorems, due to Hilbert and developed more directly by Hellinger, concerning limited linear, quadratic and hermitian forms in infinitely many numerical variables. In the present paper, which will be offered to the Transactions, Professor Moore gives definitions of the new limit and of various allied concepts, proves a number of fundamental propositions involving these concepts, and points out the sense in which various classical limits are instances of the new limit. The definition is as follows: Consider a class $\mathfrak{Q}$ of elements $q$ and a binary relation $\mathrm{k}$ on $\mathfrak{Q}$ which is reflexive, transitive and of such a nature that for every two elements $q_{1}, q_{2}$ there is an element $q_{12}$ of such a nature that $q_{12} \mathrm{~K} q_{1}$ and $q_{12} \mathrm{~K} q_{2}$. (Examples: I. $\mathfrak{Q}$ is the class of positive integers $q$; $\mathrm{K}$ is the binary relation $\geqq$ on $\mathfrak{Q}$. II. $\mathfrak{Q}$ is the class of all finite subclasses $q$ of a class $\overline{\mathfrak{P}}$ of elements $p ; \mathrm{K}$ is the binary relation $\supset$, inclusion, on $\mathfrak{Q}$, viz., for two classes $q_{1}, q_{2}, q_{1} \supset q_{2}$ denotes that every element $p$ of $q_{2}$ is an element of $q_{1}$.) Then, with respect to this relation $\mathrm{K}$, a numerically valued function $\varphi$ or $\varphi(q)$ on $\mathfrak{Q}$ has the number $a$ as limit in case for every positive number $e$ there exists an element $q_{e}$ depending on $e$ of such a nature that for every element $q \mathrm{~K} q_{e}$ it is true that $|\varphi(q)-a| \leqq e$. (Accordingly, in example I, the number $a$ is the limit in the classical sense of the numerical sequence $\varphi(1), \varphi(2), \cdots, \varphi(q), \cdots$. The limit of example II is the limit used in the definition of the integration process $J$; it is to be noted that this limit is of general reference in the sense that it has reference to no metric or other features of the class $\mathfrak{B}$ which is accordingly a (truly) general class.)

27. The differentiating machine designed by Mr. Elmendorf plots the differential curve of any given curve, and is primarily applicable in drawing the rate curve for any empirical curve. A silver reflector is mounted at the end of a bar and at right angles to it so that when the mirror is set upon the curve and turned until the image and the actual curve form a continuous line, the bar is tangent to the curve at the point in question. The lengths cut off by the bar on a vertical erected at one end of a horizontal link of constant length which is so arranged that the other end is over the center of the mirror, are plotted as ordinates of the desired differential curve. An 
account of the machine was published in the Scientific American Supplement for Feb. 12, 1916.

28. A cubic which has eight consecutive points in common with a curve $C$ at a point $P$ is called an eight-pointic cubic of $P$. One of the $\infty^{1}$ eight-pointic cubics has a double point at $P$ and is known as the eight-pointic nodal cubic. The double point tangents are, in general, distinct and form, with the inflectional line of the cubic, a triangle, which is called the canonical triangle.

The anharmonic curve which has eight consecutive points in common with $C$ at the point $P$ is called the osculating anharmonic curve. Associated with an anharmonic curve is, in general, a triangle, called the invariant triangle, which has the following property. Suppose any tangent is drawn to the curve. The anharmonic ratio of the point of tangency and the three points of intersection of the tangent with the sides of the invariant triangle is constant for all points on the curve.

Associated with every point $P$ on the curve $C$ there are six points and six lines which form the vertices and sides of the canonical triangle and the invariant triangle of the osculating anharmonic. As $P$ moves along the curve $C$ the six points will describe certain loci and the six lines will envelope certain curves. The object of Mr. Harding's paper is to study the character of these loci. The paper will be offered for publication to the Giornale di Matematiche.

29. Professor Wilczynski has shown that the theory of nonruled analytic surfaces may be based on a completely integrable system of partial differential equations of the form

$$
\begin{aligned}
& \frac{\partial^{2} y}{\partial u^{2}}+2 b \frac{\partial y}{\partial v}+f y=0, \\
& \frac{\partial^{2} y}{\partial v^{2}}+2 a^{\prime} \frac{\partial y}{\partial u}+g y=0 .
\end{aligned}
$$

In the present paper, Miss Sperry associates with each point of the surface a line through the point but not in the tangent plane of the point. In this way a congruence $L$ is determined. There are two one-parameter families of curves on the surface along which the lines of $L$ generate developables. We have called them the congruential torsal curves. On every surface 
there is a two-parameter family of curves having the property that the osculating planes of all the curves of the family passing through a point have in common the line of $L$ through that point. Their differential equation is

$$
\begin{aligned}
\frac{d^{2} u}{d t^{2}} \frac{d v}{d t}-\frac{d^{2} v}{d t^{2}} \frac{d u}{d t}+2\left\{b\left(\frac{d u}{d t}\right)^{3}-a^{\prime}\left(\frac{d v}{d t}\right)^{3}\right\}+ \\
2\left\{p_{1}\left(\frac{d u}{d t}\right)^{2} \frac{d v}{d t}+p_{2} \frac{d u}{d t}\left(\frac{d v}{d t}\right)^{2}\right\}=0,
\end{aligned}
$$

where $p_{1}$ and $p_{2}$ depend upon the choice of $L$.

Conversely the integral curves of an equation of this type have the property that the osculating planes of all the curves of the family which pass through a point form a pencil. These curves we have called the congruential union curves. If $L$ is the congruence of surface normals the torsal curves are lines of curvature and the union curves are geodesics. If $L$ is the congruence of directrices of the second kind the torsal curves are directrix curves.

A necessary and sufficient condition that the union curves be plane is that they be torsal curves, a generalization of a well known theorem concerning geodesics and lines of curvature.

30. With every point $P$ of a non-ruled surface $S$ Professor Wilczynski has associated a pair of straight lines; one of these lines lies in the tangent plane and is called the directrix of the first kind, while the other pierces the surface at $P$ and is called the directrix of the second kind. The two families of developables of the two congruences thus associated with $S$ are determined by one and the same net of curves, the directrix curves on $S$.

In this paper Dr. Yeaton obtains the conditions under which the focal sheets of the directrix congruence of the second kind degenerate into curves. Limiting the discussion to surfaces whose directrix curves form a conjugate net, he shows that if this net is not degenerate, the congruence in question may be linear. The surfaces thus determined are projectively equivalent to the surface

$$
z=\frac{1}{4} x y\left(\log _{e} \frac{x}{y}\right)^{2}
$$


On any surface the two families of asymptotic curves are projectively equivalent, each lies on a quadric and is identically self-dual; the directrix curves are plane curves and one of the two families consists of conics. A non-degenerate quadric and a straight line, which is not a ruling of the quadric, constitute the focal surface of the directrix congruence of the first kind. The finite equations of the various associated loci are obtained.

\author{
ARNold Dresden, \\ Secretary of the Section.
}

\title{
NOTE ON FUNCTIONS OF SEVERAL COMPLEX VARIABLES.
}

BY PROFESSOR WILLIAM F. OSGOOD.

(Read before the Amerioan Mathematical Society, April 29, 1916.)

THE object of the present note is at once to extend the scope of a fundamental theorem of the theory of analytic functions of several complex variables and to simplify its proof.*

Definition.-Let $S$ be the cylindrical region $\left(S_{1}, \cdots, S_{n}\right)$,

$S_{k}$ :

$$
\left|z_{k}\right|<r_{k}
$$$$
(k=1, \cdots, n) \text {; }
$$

let $\Sigma$ be the region $\left(\Sigma_{1}, \cdots, \Sigma_{n}\right)$,

$$
\begin{gathered}
\left|z_{j}\right|<h_{j}<r_{j} \\
\left|z_{k}\right|<r_{k}
\end{gathered}
$$$$
(j=1,2) \text {; }
$$$$
\Sigma_{k} \text { : }
$$$$
(k=3, \cdots, n) \text {; }
$$

and let $T$ be the region whose points are interior to $S$, but exterior to $\Sigma$ :

$$
T=S-\Sigma \cdot \dagger
$$

Theorem. Let $f\left(z_{1}, \cdots, z_{n}\right)$ be analytic throughout the region T. Then $f\left(z_{1}, \cdots, z_{n}\right)$ admits analytic continuation throughout $S$.

* The theorem was given by Kistler, "Ueber Funktionen von mehreren komplexen Veränderlichen," $\S 7$, Basel, 1905, for the case that the excepted points lie on a finite number of analytic manifolds, each of $n-2$ complex dimensions, and was proven by means of $n$-fold integrals.

$\dagger$ This symbolic form is suggestive, but not quite accurate, since it would assign to $T$ certain of its boundary points, and $T$ consists only of interior points. 